\title{
Matrizes poliméricas para encapsulação de bioinseticidas
}

\author{
Polymeric matrixs for bioinsecticide encapsulation
}

Carla Moraes Lêdo ${ }^{*}$, Márcia Nieves Carneiro-da-Cunha',3, José de Paula Oliveira ${ }^{4}$,

José Manoel Wanderley Duarte-Neto ${ }^{4}$, Ana Lúcia Figueiredo Porto²

'Departamento de Biologia, Autarquia Educacional de Serra Talhada, Av. Afonso Magalhães, 380, CEP 56912 380, Serra Talhada, PE, Brasil 2Departamento de Morfologia e Fisiologia Animal, Universidade Federal Rural de Pernambuco, Rua Dom Manoel de Medeiros, s/n, Dois Irmãos, CEP 52171-900, Recife, PE, Brasil

${ }^{3}$ Bolsista na modalidade BFP da Fundação da Amparo a Ciência e Tecnologia do Estado de Pernambuco (FACEPE)

${ }^{4}$ Laboratôrio de Biotecnologia, Instituto Agronômico de Pernambuco,

Av. General San Martin, 1371, CEP

50761-000, Bongi, Recife, PE, Brasil

* autor correspondente

《 carlaledo@yahoo.com.br
RESUMO: Os bioinseticidas apresentam uma alta suscetibilidade a fatores ambientais, e a busca por técnicas que protejam esses princípios ativos vem sendo proposta. O objetivo do trabalho foi apresentar a técnica de microencapsulação em biomoléculas inseticidas, a fim de promover a proteção contra estresses ambientais, assim como os principais polímeros utilizados no processo. A pesquisa resultou em poucos trabalhos na microencapsulação de biomoléculas para este fim. Na maior parte deles, a técnica utilizada foi a de spray drying, e os materiais de microencapsulamento foram os polímeros de carboidratos, destacando o alginato. Essa técnica apresenta-se eficiente para conservação, durabilidade de prateleira e proteção a fatores ambientais.

PALAVRAS-CHAVE: Microencapsulação, polímeros, inseticidas biológicos

\begin{abstract}
Bioinsecticides are highly susceptible to environmental factors, therefore requiring techniques that protect these active ingredients. This work aimed to present the microencapsulation technique in insecticidal biomolecules to promote protection against environmental stresses, as well as the main polymers used in the process. The research resulted in few studies on microencapsulation of biomolecules for this purpose. In most of them, used the spray drying technique, with the microencapsulation material being carbohydrate polymers, with alginate standing out. This technique is efficient for conservation, shelf life and protection from environmental factors.
\end{abstract}

KEYWORDS: Microencapsulation, polymers, biological insecticides

\section{Introdução}

Os bioinseticidas são produtos que utilizam em sua formulação organismos vivos ou derivados, que atuam como agentes de controle biológico, e podem ser classificados como microbiológicos quando constituídos por bactérias, fungos ou vírus, ou macrobiológicos quando trazem em sua composição insetos, ácaros ou nematoides (BRASIL, 2019). Distribuídos em área e valor, respectivamente de $20 \%$ e $11 \%$ nos macrobiológicos e de $80 \%$ e $89 \%$ nos microbiológicos (BORSARI; CLAUDINO, 2019), o uso desses bioinsumos tem se tornado cada vez mais popular como alternativas aos agroquímicos por uma variedade de razões, incluindo o surgimento de pragas resistentes a muitos inseticidas sintéticos; o aumento na demanda pública por frutas e vegetais cultivados organicamente; as regulamentações sobre limites máximos de resíduos desses produtos na produção e as preocupações sobre o impacto ambiental e efeitos nocivos a organismos não alvo associados ao uso de inseticidas sintéticos (DARA, 2017; GLARE; O'CALLAGHAN, 2019; GLARE et al., 2012; LACEY et al., 2015; PREININGER et al., 2018). 
Os bioinseticidas desempenham um importante papel nos programas de manejo integrado de pragas, porque possuem atividade residual curta, permitindo que os trabalhadores agrícolas reentrem rapidamente nos campos tratados, pois apresentam baixa toxicidade humana e ambiental e efeitos não direcionados mínimos (CHANDLER et al., 2008; COPPING; MENN, 2000; SPORLEDER; LACEY, 2013). Além disso, muitos bioinseticidas foram aprovados pelo Organic Materials Review Institute (OMRI), permitindo que sejam usados em sistemas de produção de culturas orgânicas e convencionais.

Contudo, os agroquímicos são frequentemente mais baratos (BALE et al., 2008; THAKORE, 2006), mais fáceis de aplicar (PREININGER et al., 2018), armazenados por mais tempo (DUNHAM, 2015), com ação mais rápida (PREININGER et al., 2018) e geralmente considerados mais eficazes, quando comparados com os bioinseticidas (ARTHURS e DARA, 2019). Dessa forma, torna-se imprescindível o desenvolvimento de novas formulações e tecnologias de aplicação de bioinseticidas, com o intuito de contornar problemas, diminuir os custos de produção desses produtos e torná-los mais competitivos no mercado.

Nesse contexto, a utilização de técnicas de encapsulação na escala micro e nanométrica vem sendo sugerida para produção de bioinseticidas, com o objetivo de aumentar estabilidade e proteger os agentes ativos dessas formulações de fatores ambientais, tais como: radiação ultravioleta, chuva, $\mathrm{pH}$, temperatura e fisiologia da folhagem, que em campo prejudicam a eficácia e diminuem persistência das formulações (AUGUSTIN; HEMAR, 2009; BRAR et al., 2006; CAM; ICYER; ERDOGAN, 2014; MCCLEMENTS; LI, 2010; PEREZ-MASIA et al., 2015)

Os microencapsulados podem ser produzidos a partir de materiais biodegradáveis e liberar lentamente as moléculas funcionais que eles contêm, o que reduz as aplicações frequentes. Além disso, são em tamanhos pequenos com grande capacidade de carga e área de superfície, e maior estabilidade e solubilidade quando comparados com seus equivalentes volumosos (ALARCÓN-ALARCÓN et al., 2018; ANNUNZIATA et al., 2020; BEZERRA, et al., 2016; ESSER-KAHN, et al., 2011; TOMAZELLI et al., 2018; ZAITOON et al., 2021). Assim, dada a crescente demanda global por alimentos e a urgência em desenvolver estratégias sustentáveis para o controle de pragas, uma das soluções alternativas é o uso de bioinseticidas microencapsulados (FERNÁNDEZ-PÉREZ et al., 2014).

Diante do exposto, este trabalho propõe uma breve revisão das principais matrizes utilizadas para encapsulamento de substâncias biológicas com ação inseticida, mostrar a eficiência para preservar o princípio ativo da ação de fatores ambientais e controlar a administração e liberação das doses, assim como a técnica utilizada para encapsular.

\section{Metodologia}

A pesquisa foi elaborada utilizando as bases de busca Scopus, ScienceDirect e SciELO; o levantamento da literatura disponível sobre o tema foi realizado a partir dos descritores: "Microencapsulation AND Bioinsecticide" no período de outubro de 2020 a março de 2021. Foram considerados apenas os artigos de pesquisa que utilizaram as matrizes poliméricas (simples ou combinadas) para o encapsulamento de bioinseticidas, artigos científicos que avaliaram o encapsulamento de agroquímicos foram descartados, nenhuma restrição foi utilizada referente ao ano das publicações, apenas artigos de pesquisa foram selecionados, revisões de literatura ou outros documentos não foram considerados para esta pesquisa. Após a seleção dos artigos de interesse, foi realizada uma breve descrição dos trabalhos encontrados, com ênfase para a biomolécula com atividade inseticida, o tipo de material encapsulante, a técnica de encapsulação utilizadas e os principais resultados obtidos.

\section{Resultados e Discussão}

\section{Bioinseticidas microencapsulados}

A microencapsulação é um processo de empacotamento de materiais sólidos, líquidos ou gasosos em cápsulas extremamente pequenas, as quais podem liberar o conteúdo de forma controlada e sob condições específicas. O uso dessas técnicas vem sendo sugerido como um método promissor para a proteção de compostos bioativos. O mecanismo de proteção ocorre por causa da formação da cápsula, cobertura ou parede pelo material encapsulante, que envolvem o material encapsulado denominado de recheio ou núcleo (BARRETO et al., 2015; XING et al., 2014).

A utilização de técnicas de microencapsulação na formulação de bioinseticidas vem sendo proposta por diferentes autores como uma ferramenta viável que tem como objetivo a diminuição da biodegradação e alta instabilidade frente a fatores ambientais desses bioprodutos, e consequentemente aumentar sua eficácia e persistência em campo. A Tabela 1 apresenta os tipos de bioinseticidas, as técnicas de microencapsulação e as principais matrizes poliméricas utilizadas no revestimento de bioinseticidas de acordo com a literatura.

Técnicas para microencapsulação de bioinseticidas

Várias técnicas têm sido empregadas na elaboração de microcápsulas, tais como a secagem por atomização (spray drying), coacervação, emulsão, extrusão, recobrimento em leito fluidizado, lipossomas e complexação por inclusão (SOHAIL et al., 2011). A seleção do método a ser utilizado depende do tamanho desejado da microcápsula e da aplicação que será dada a ela, do mecanismo de liberação e das propriedades físico-químicas, tanto do material ativo quanto do agente encapsulante (ASSUNÇÃO, 2014; COOK et al., 2012). A maneira como ocorre a liberação do bioativo presente no núcleo da microcápsula é ponto crucial no sucesso da ação da micropartícula em seu uso final. Geralmente, a escolha da técnica da encapsulação foca o controle da excreção da substância envolvida, tendo a finalidade de definir o modo de como ela será liberada, os locais onde o bioativo precisa ser preservado ou liberado para começar agir, o tempo em que ele levará para começar a ser liberado e a duração da mesma (CASTRO; RODRIGUES, 2003).

Considerando a microencapsulação de bioinseticidas, a técnica mais utilizada descrita na literatura é secagem por atomização (spray drying), na qual um produto líquido é atomizado em ar 
Tabela 1. Bioinseticidas microencapsulados.

\begin{tabular}{|c|c|c|c|}
\hline Tipo de bioinseticida & Técnica de microencapsulação & Material de Parede & Autor/Ano \\
\hline \multirow{8}{*}{ Bacillus thuringiensis } & \multirow{3}{*}{ Spray drying } & Amido & \multirow{3}{*}{ Eski et al. (2019) } \\
\hline & & Goma-arábica & \\
\hline & & Maltodextina & \\
\hline & $\begin{array}{l}\text { Deposição alternada camada- } \\
\text { camada (layer-by-layer, LBL) }\end{array}$ & $\begin{array}{l}\text { Quitosana; Alginato e } \\
\text { Carboximetilcelulose }\end{array}$ & He et al. (2017) \\
\hline & Emulsão / gelificação interna & Óleo de milho, alginato e tween & Barrera-Cortés et al. (2017) \\
\hline & Spray drying & Amido de amaranto & Rodríguez et al. (2015) \\
\hline & Emulsão / gelificação interna & Alginato & García-Gutiérrez et al. (2011) \\
\hline & Emulsão / gelificação interna & Amido, gelatina e alginato de sódio & Khorramvatan et al. (2014) \\
\hline \multirow{2}{*}{$\begin{array}{l}\text { Extrato de Azadirachta } \\
\text { indica }\end{array}$} & \multirow{2}{*}{ Spray drying } & Goma-arábica & \multirow{2}{*}{ Bezerra et al. (2020) } \\
\hline & & Maltodextrina & \\
\hline Espinosade & Spray drying & $\begin{array}{l}\text { Quitosana }(\mathrm{CH}) \text { e lignossulfonato de } \\
\text { sódio (SL) }\end{array}$ & Pérez-Landa et al. (2021) \\
\hline Trichoderma harzianum & Spray drying & Maltodextrina e goma-arábica & Muñoz-Celaya et al. (2012) \\
\hline Beauveria bassiana & Spray drying & $\begin{array}{c}\text { Hidroxipropilmetilcelulose } \\
\text { quitosana, dextrina, leite desnatado } \\
\text { e polivinilpirrolidona }\end{array}$ & Liu e Liu (2009b) \\
\hline $\begin{array}{c}\text { Metarhizium anisopliae } \\
\text { MA126 }\end{array}$ & Spray drying & $\begin{array}{c}\text { Alginato de sódio, } \\
\text { hidroxipropilmetilcelulose (HPMC) } \\
\text { e quitosana }\end{array}$ & Liu e Liu (2009a) \\
\hline proteina $r V P r 1$ & Gelificação iônica & Alginato de sódio & Richards et al. (2015) \\
\hline \multirow{2}{*}{$\begin{array}{c}\text { Nucleopoliedrovírus } \\
\text { de Anagrapha falcifera } \\
\text { (Kirby) }\end{array}$} & Spray drying & Lignina & Behle et al. (2003) \\
\hline & Spray drying & $\begin{array}{l}\text { Farinhas de milho, lignina e } \\
\text { sacarose }\end{array}$ & Tamez-Guerra et al. (2002) \\
\hline
\end{tabular}

quente para obtenção de um pó, que em condições ideais este pó obtido apresenta como características boa qualidade, baixa atividade de água, facilidade de armazenamento e estocagem, além de proteger o material ativo contra reações indesejáveis (PEREIRA et al., 2018). Eski et al. (2019) e Rodríguez et al. (2015) empregaram esta técnica para encapsular bactéria entomopatogênica Bacillus thuringiensis (Bt), que é o pesticida microbiano mais utilizado, sendo por isso considerado o principal bioinseticida. Contudo, quando aplicado em superfícies foliares, as condições ambientais como alta temperatura, radiação UV e precipitação podem reduzir ou inativar sua atividade (HE et al., 2017). Em ambos os estudos, os autores observaram que a microencapsulação conferiu às formulações uma maior estabilidade em comparação com formulados não encapsulados.

Da mesma forma, o espinosade, um bioinseticida obtido a partir da fermentação do actinomiceto Saccharopolyspora spinosa, foi microencapsulado através da técnica de spray drying para obtenção um sistema fotoprotetor, visto que sua atividade, assim como a do Bt, também pode ser afetada pela fotodegradação (PÉREZ-LANDA et al., 2021). Experimentos conduzidos visando avaliar a fotodegradação do espinosade microencapsulado e não microencapsulado demonstraram uma taxa de degradação $25 \%$ menor para o bioinseticida microencapsulado após $24 \mathrm{~h}$ de exposição à luz ultravioleta. A técnica de spray drying também foi utilizada para microencapsular os fungos entomopatogênicos Trichoderma harzianum (MUÑOZ-CELAYA et al., 2012),
Metarhizium anisopliae MA126 (LIU; LIU, 2009a) e Beauveria bassiana (LIU; LIU, 2009b), o nucleopoliedrovírus de Anagrapha falcifera (BEHLE et al., 2003; TAMEZ-GUERRA et al., 2002) e os inseticidas biodegradáveis à base de Azadirachta indica A. Juss. (Popularmente conhecida como "nim") ( BEZERRA et al., 2020). Segundo Bezerra et al. (2020), muitos fatores podem afetar o desempenho de secagem, sejam extrínsecos (umidade e temperatura ambiente) ou intrínsecos (viscosidade, transição vítrea). Desta forma, é na otimização do processo que a análise dessas variáveis deve ser considerada.

A técnica de emulsão/gelificação iônica interna foi sugerida por Barrera-Cortés et al. (2017), Khorramvatan et al. (2014) e García-Gutiérrez et al. (2011) para obter formulados de Bt mais estáveis. É uma técnica rápida, simples e de baixo custo, cuja formação das microcápsulas baseia-se na capacidade de polietrólitos de fazerem ligações cruzadas na presença de contraíons, formando uma matriz tridimensional (HOLKEM; CODEVILLA; DE MENEZES, 2015).

He et al. (2017) realizaram o microencapsulamento de preparações contendo cristais de Bt através da técnica de deposição alternada camada-camada (layer-by-layer, LBL), que consiste na deposição um substrato de várias camadas de polieletrólitos com cargas superficiais diferentes entre si. Os autores utilizaram quitosana e alginato alternadamente, até a obtenção de dez camadas; após a deposição da quinta camada de polímero, foi observada uma melhora na dispersão dos 
cristais em solução. A análise da morfologia das microcápsulas utilizando o MEV indicou que o biopolímero se depositou na superfície dos cristais, formando uma microcápsula bipiramidal, e o tamanho era de cerca de $0,6 \times 1,2 \mu \mathrm{m}$, tamanho adequado para ingestão de larvas.

A escolha do método mais adequado depende do tipo do material ativo e sua aplicação. Para essa escolha, tem que ser analisada a estabilidade do princípio ativo a métodos químicos ou físicos e custo para o procedimento, assim como a morfologia capsular desejada. Contudo, o método mais utilizado é o spray drying, pois este processo é econômico, rápido, adequa-se facilmente à escala industrial e permite encapsular materiais instáveis, uma vez que o tempo de secagem é reduzido.

\section{Matrizes poliméricas utilizadas como material encapsulante}

A escolha do material encapsulante deve levar em consideração uma série de fatores, como propriedades físicas e químicas do núcleo (porosidade, solubilidade, etc.) e da parede (viscosidade, propriedades mecânicas, transição vítrea, capacidade de formação de filme, etc.), mecanismo de controle e fatores econômicos (AGNIHOTRI et al., 2012; ECKERT et al., 2017, ESKI et al., 2017; RODRÍGUEZ et al., 2015; SHOKRI et al., 2015). A estabilização deve proteger o material do núcleo da degradação, a fim de evitar a modificação de algumas características e permitir manuseio mais fácil, fornece barreiras entre os materiais bioativos sensíveis, e a possibilidade de controlar a taxa de liberação (DA SILVA et al., 2016).

A eliminação do núcleo pode ocorrer de maneira mecânica quando algo entra em atrito com o microencapsulado e rompe o revestimento da partícula, por dissolução do encapsulante em solvente de interesse, dependendo da temperatura que muda o estado da matéria ou por meio de alterações químicas. O material utilizado na envoltura da micropartícula e o modo de liberação definido para tal irão definir a velocidade e a quantidade do produto liberado, o que também depende de qual será o uso da substância (CANDIAGO et al., 2018).

De acordo com Suave et al. (2006), os materiais mais utilizados como encapsulantes incluem: carboidratos (amido, dextrinas, açúcar, xarope de milho, celuloses), gomas (goma arábica, alginato de sódio, carragena), lipídeos (cera, parafina, triestearina, ácido esteárico, monoglicerídeos e diglicerídeos, óleos e gorduras hidrogenadas, poliésteres naturais poli(hidroxialcanoatos), poli(3-hidroxibutirato) $\mathrm{P}(3 \mathrm{HB})$, poli(3-hidroxivalerato) $\mathrm{P}(3 \mathrm{HV}) \mathrm{e}$ seus copolímeros), polímeros sintéticos (poli(D, L-ácido láctico) (PDLA), poliacrilatos, copolímeros de polietilenoco-propileno, poli(e-caprolactona) (PCL)), proteínas (glúten, caseína, gelatina, albumina). Entretanto, nos últimos anos, intensificou-se o uso de polímeros naturais, devido às características inerentes a esses materiais, pois são atóxicos, de baixo custo e biodisponíveis. Além disso, os polímeros são biodegradáveis, permitindo a entrega contínua do agente ativo com baixo dano ambiental (CAMPOS et al., 2019).

Entre os polímeros biodegradáveis encontram-se os polissacarídeos ou proteínas de origem animal ou vegetal, que podem ser utilizados como cobertura para as microcápsulas. Os polissacarídeos mais utilizados são os amidos, celulose, gomas e pectinas. Entre os biopolímeros compostos por proteínas, encontram-se: colágeno, gelatinas, caseínas e queratina (GALUS; KADZINSKA, 2015; MCMILLIN, 2017). Outro ponto a ser considerado é o emprego de mais de um polímero como material encapsulante, em geral, são utilizadas associações de dois ou mais tipos de polímeros em diferentes concentrações. A Tabela 2 apresenta os principais polímeros utilizados em técnicas de microencapsulação e suas características.

Dentre as matrizes poliméricas utilizadas no encapsulamento de bioinseticidas, o alginato de sódio tem sido mais frequentemente empregado. Esse polímero permite a formação de géis rapidamente em presença de íons cálcio, sem alterações drásticas de temperatura, pH e pressão osmótica, preservando a atividade e a viabilidade dos micro-organismos microencapsulados (CARVALHO et al., 2006). Como o observado por Khorramvatan et al. (2014), quando avaliaram o efeito dos polímeros gelatina, amido e alginato de sódio na estabilidade de formulações microencapsuladas de Bacillus thuringiensis subsp. kurstaki (Bt-KD2), após exposição à radiação ultravioleta UVB $(385 \mathrm{~nm})$ por 7 dias houve uma diminuição da viabilidade dos esporos de 28,35 , 40 e $60 \%$ para alginato de sódio, amido, gelatina e esporos livres, respectivamente. $\mathrm{O}$ alginato de sódio apresentou a maior preservação de viabilidade e a proteção de polímero atingiu um estado estacionário após $120 \mathrm{~h}$, resultados que evidenciam o efeito fotoprotetor desses polímeros. Corroborando esses resultados, Richards et al. (2015) confirmaram a eficácia de encapsulação e liberação da proteína rVPr1 (uma proteína imunossupressora de inseto e um bioinseticida potencial), para larvas de Lacanobia oleracea. Eles observaram que microesferas de alginato se quebram rapidamente nas condições alcalinas do intestino dos insetos e liberam seu conteúdo, passando através do intestino em 2-3 h. E que as microesferas podem ser armazenadas em uma solução de $\mathrm{CaCl}_{2}$ por até 24 dias sem vazamento.

Corroborando esses resultados, He et al. (2017) utilizaram os polímeros quitosana, alginato e carboximetilcelulose na microencapsulação de cristais parassporais sintetizados por Bacillus thuringiensis. Tais autores relatam a eficiência do processo de encapsulação, visto que as preparações microencapsuladas apresentaram toxicidade larvicida equivalente à da forma não encapsulada. Os cristais microencapsulados foram protegidos do efeito da alta temperatura e dessecação. Dentre os polímeros avaliados pelos pesquisadores, a combinação da quitosana com alginato forneceu a melhor proteção a temperatura e contra a dessecação. Os resultados indicam que a proteção da microcápsula pode aumentar a eficácia do bioinseticida no controle de pragas.

Um tipo de agente microencapsulante pode não ter todas as propriedades necessárias, portanto, combinações de diferentes agentes microencapsulantes podem ser adotadas para uma melhor produção de microcápsulas. A goma-arábica e a maltodextrina foram utilizadas no microencapsulamento do extrato de Azadirachta indica (um extrato vegetal que possui ação inseticida), visando à liberação controlada do bioinseticida para o meio ambiente, reduzindo seu uso. Os resultados apresentados para cinética de liberação do bioinseticida mostraram que não houve liberação imediata e ocorreu um aumento gradual na concentração de azadiractina ao longo do tempo (liberação 
Tabela 2. Principais polímeros utilizados na microencapsulação.

\begin{tabular}{|c|c|}
\hline Polímero & Característica /Vantagem do uso \\
\hline Alginato & $\begin{array}{l}\text { É um polissacarídeo linear obtido a partir de algas marrons ou bactérias, compostos por resíduos dos ácidos } \beta \text {-D- } \\
\text { manurônico e } \alpha \text {-Lgulurônico na forma de sal de sódio unidos por ligações glicosídicas e distribuídos em diferentes } \\
\text { proporções ao longo da cadeia. }\end{array}$ \\
\hline Quitosana & $\begin{array}{l}\text { Pode polimerizar através de uma formação de ligações cruzadas na presença de ânions e poliânions, sendo } \\
\text { preferencialmente utilizado como revestimento (MORTAZAVIAN et al., 2008). }\end{array}$ \\
\hline Goma-arábica & $\begin{array}{l}\text { Apresenta 5\% de proteína (RIBEIRO; SERAVALLI, 2007), sendo esta fração de proteína responsável pela } \\
\text { propriedade de emulsificação da goma, o que a torna adequada para a encapsulação por spray drying (SHAHIDI; } \\
\text { HAN, 1993). }\end{array}$ \\
\hline Maltodextrina & $\begin{array}{l}\text { São consideradas bons agentes encapsulantes por apresentarem baixa higroscopicidade, boa solubilidade e baixa } \\
\text { viscosidade (KOÇ et al., 2015; ANEKELLA; ORSAT, 2013). }\end{array}$ \\
\hline Pectina & $\begin{array}{l}\text { Devido às características eletrostáticas e de formação de géis, apresenta-se como um bom material de parede } \\
\text { (ABERKANE; ROUDAUT; SAUREL, 2014). }\end{array}$ \\
\hline Goma xantana & $\begin{array}{l}\text { Devido a suas propriedades reológicas, permite a formação de soluções viscosas a baixas concentrações }(0,05- \\
1,0 \% \text { ) e estabilidade em ampla faixa de pH e temperatura (FONTANIELLA et al., 2002). }\end{array}$ \\
\hline $\begin{array}{l}\text { Carboximetil } \\
\text { celulose }\end{array}$ & $\begin{array}{c}\text { Apresenta resistência ao ácido gástrico e às propriedades de solubilidade intestinais, que permitem sua utilização } \\
\text { na liberação controlada (KAMEL et al., 2008). }\end{array}$ \\
\hline
\end{tabular}

de $100 \%$ da azadiractina ocorreu com 20h). Além disso, a microcápsula sintetizada com os polímeros, também apresentou um efeito fotoprotetor, com um tempo de fotodegradação de $50 \%$ da azadiractina de 21,07 dias, com apenas $38,17 \%$ de fotodegradação após 15 dias (BEZERRA et al., 2020).

Essa mesma matriz (maltodextrina e goma-arábica) foi empregada por Muñoz-Celaya et al. (2012) para a microencapsulação de conídios do fungo entomopatogênico Trichoderma harzianum. Foi observada uma sobrevivência de $86 \%$ após a pulverização, e após 8 semanas essa sobrevivência foi de $40 \%$ após estocagem a $4^{\circ} \mathrm{C}$, sendo 11 e 330 vezes maiores do que aquelas de conídios não encapsulados, respectivamente. Barrera-Cortés et al. (2017), usando óleo de milho, solução de alginato de sódio e surfactante para emulsionar os cristais, constataram a diminuição das microcápsulas, 8,5 $\pm 1 \mathrm{~nm}$ e uniformidade dos diâmetros. Além disso, mostrou alta resistência à irradiação extrema $(2,9 \pm$ $0,5 \times 108 \mathrm{erg}$ ) de comprimento de onda longo (365 nm). Esses resultados demonstram a eficiência dos agentes encapsulantes na proteção contra fatores ambientais.

Desta forma, o critério para a seleção do material encapsulante depende de suas propriedades, tais como compatibilidade dos materiais da cápsula, a estrutura dos agentes encapsulantes e os aspectos econômicos e de processamento envolvidos. A escolha de um agente encapsulante depende também do nível de toxicidade, estabilidade, eficiência, grau de proteção e propriedades microscópicas das microcápsulas produzidas.

\section{Bioatividade dos bioinseticidas microencapsulados}

A avaliação da eficiência das formulações de bioinseticidas microencapsulados foram conduzidas frente a diferentes insetos (pragas agrícolas) com o objetivo de estudar a influência da microcápsula sobre a atividade inseticida. Entretanto, nos resultados demonstrados por West (1997), trabalhando com micropartículas quitosana $(\mathrm{CH})$ e lignossulfonato de sódio (SL) contendo o espinosade fotossensível (SP), a quitosana funcionou para manter a estrutura e promover a liberação de inseticida, que foi de $70 \%$ até $6 \mathrm{~h}$, apresentando um tempo de liberação eficiente no estômago dos insetos, adequando-se ao uso como estratégia para reduzir impactos negativos ao meio ambiente nas práticas agronômicas.

Os bioensaios realizados por He et al. (2017) utilizando formulados microencasulados de Bt demonstraram que as preparações tinham toxicidade larvicida para Ostrinia furnacalis, equivalente à forma não encapsulada, apresentando resistência após o tratamento em $50^{\circ} \mathrm{C}$, e a quantidade de proteínas após o tratamento em radiação UV por $0,5 \mathrm{~h}$ permaneceu a mesma. Da mesma forma, Eski et al. (2017), após a microencapsulação dos cristais utilizando a maltodextrina, observou o índice de mortalidades de 93\% para larvas de Spodoptera exigua após 10 dias de tratamento, mostrando a eficiência na estabilidade e proteção dos cristais.

Biopesticidas à base de óleo essencial podem ser usados como uma alternativa protetora para ataques de pragas. Desta forma, Putri et al. (2019) microencapsularam o óleo essencial Eugenol, presente na planta de cravo-da-índia (Syzygium aromaticum), através da técnica de micela de caseína, e caseína junto com o fosfato de cálcio pode formar uma espécie de partículas coloidais dispersas, chamadas micelas (Phadungath, 2005). A porcentagem de eugenol encapsulado com sucesso foi de $87,99 \%$, e os testes foram realizados em Apis mellifera, após 24 h após o encapsulamento, e houve uma diminuição da atividade; contudo, apresentou toxicidade. Já Sharma e Goel (2018) trabalharam com a goma da acácia para microencapsular dois óleos, o de eucalipto e o de cedro (materiais de núcleo), através de coacervação simples. Os dois apresentaram atividade repelente para Lepisma saccharina semelhante aos não encapsulados, após a microencapsulação.

Os trabalhos descritos apresentaram pouca ou nenhuma perda de atividade após o encapsulamento, e os resultados demonstraram efetiva resistência aos fatores ambientais. 


\section{Conclusão}

A busca por substâncias naturais para o controle de pragas cresceu muito nos últimos anos, reflexo de uma conscientização de proteção do meio ambiente e por uma vida mais saudável, contudo, essas substâncias são facilmente degradadas por fatores ambientais, tornando inviáveis sua comercialização. Mesmo apresentando poucos trabalhos disponíveis, a partir da metodologia utilizada, o trabalho apresentou várias técnicas eficientes na proteção dos bioinseticidas contra os fatores ambientais, como, radiação UV, dessecação e durabilidade. Foram encontrados trabalhos de microencapsulação de diversos agentes bioinseticidas, como toxinas microbiológicas e óleos essenciais. A toxina produzida por $\mathrm{Bt}$ se destacou em número de trabalhos dessa natureza. Já dentre os materiais utilizados nas técnicas de encapsulação, o alginato aparece em destaque, pois tem sido amplamente utilizado, pois as microesferas feitas com esse material podem ser produzidas em uma variedade de tamanhos, são palatáveis e não tóxicas para as larvas de insetos. E dentre as técnicas mais utilizadas para encapsulação, a mais utilizada foi a de Spray drying. Esse conceito requer mudanças de paradigmas no desenvolvimento de novos produtos, aplicando as tecnologias tradicionais, aliadas aos novos métodos de conservação, preservando o máximo possível os componentes bioativos dos bioinseticidas, sem que eles percam sua eficiência e, ainda, aumentando sua durabilidade em campo.

\section{Agradecimentos}

Ao Conselho Nacional de Desenvolvimento Científico e Tecnológico (CNPq) (processo: 426192/2018-4) e a Fundação de Amparo à Pesquisa (FACEPE) (processo: BPV-007-5.01/19, BFP-0158-5.01/19) e a Autarquia Educacional de Serra Talhada/PE.

\section{Referências}

Aberkane, L.; Roudaut, G.; Saurel, R. Encapsulation and oxidative stability of pufa-rich oil microencapsulated by spray drying using pea protein and pectin. Food and Bioprocess Technology, New York, v. 7, n. 5, p. 1505-1517, 2014.

Agnihotri, N. et al. Microencapsulation - a novel approach in drug delivery. Indo Global Journal of Pharmaceutical Sciences, India, v. 2, n. 1, p. 1-20, 2012.

Alarcón-Alarcón, C. et al. Protection of astaxanthin from photodegradation by its inclusion in hierarchically assembled nano and microstructures with potential as food. Food Hydrocolloids, Oxford, v. 83, p. 36-44, 2018. http://dx.doi.org/10.1016/j.foodhyd.2018.04.033.

Anekella, K.; Orsat, V. Optimization of microencapsulation of probiotics in raspberry juice by spray drying. Lebensmittel-Wissenschaft + Technologie, v. 50, n. 2, p. 17-24, 2013.

Annunziata, et al. Microencapsulation as a tool to counteract the typical low bioavailability of polyphenols in the management of diabetes. Food and Chemical Toxicology, Oxford, v. 139, 2020. http://dx.doi.org/10.1016/j.fct.2020.111248.

Arthurs, S.; Dara, S. K. Microbial biopesticides for invertebrate pests and their markets in the United States. Journal of Invertebrate
Pathology, San Diego, v. 165, p. 13-21, 2019. http://dx.doi. org/10.1016/j.jip.2018.01.008.

Assunção, L. S. Estudo prospectivo sobre encapsulamento de compostos bioativos. Revista Geintec - Gestão, Inovação e Tecnologias, v. 4, p. 1382-1391, 2014.

Augustin, M. A.; Hemar, Y. Nano - and micro-structured assemblies for encapsulation of food ingredientes. Chemical Society Reviews, Aracaju, v. 38, p. 902-912, 2009. http://dx.doi.org/10.1039/b801739p.

Behle, R. W.; Tamez-guerra, P.; Mcguire, M. R. Field activity and storage stability of Anagrapha falcifera Nucleopolyhedrovirus (AfMNPV) in spray-dried lignin-based formulations. Journal of Economic Entomology, Lanham, v. 96, p. 1066-1075, 2003. http:// dx.doi.org/10.1093/jee/96.4.1066.

Bale, J. S.; Van Lenteren, J. C.; Bigler, F. Biological control and sustainable food production. Philosophical. Transactions B, v. 363, p. 761-776. 2008. https://doi.org/10.1098/ rstb.2007.2182.

Barrera-Cortés, J. et al. Reducing the microcapsule diameter by micro-emulsion to improve the insecticidal activity of Bacillus thuringiensis encapsulated formulations. Biocontrol Science and Technology, Oxford, v. 27, p. 42-57, 2017.

Barreto, A. R. et al. Coating materials used in the microencapsulation of probiotics. Ciência e Natura, Santa Maria, v. 37, p. 164-174, 2015.

Bezerra, D. G. et al. Microencapsulated extracts from Azadirachta indica seeds: Acquisition, characterization, and use in controlling Helicoverpa armigera. Drying Technology, New York, v. 39, p. 1-14, 2020. http://dx.doi.org/10.1080/07373937.2020.1745823.

Bezerra, et al. Controlled release of microencapsulated citronella essential oil on cotton and polyester matrices. Cellulose, London, England, v. 23, p. 1459-1470, 2016

Brar, K. S. et al. Recent advances in downstream processing and formulations of Bacillus thuringiensis based biopesticides. Process Biochemistry, London, v. 41, n. 2, p. 323-342, 2006.

Borsari, A. P.; Claudino, M. Mercado e percepção do produtor brasileiro. AgroANALYSIS, v. 38, p. 32-37, 2019.

Cam, M.; Icyer, N. C.; Erdogan, F. Pomegranate peel phenolics: microencapsulation, storage stability and potential ingredient for functional food development. Lebensmittel-Wissenschaft + Technologie, v. 55, p. 117-123, 2014. http://dx.doi.org/10.1016/j. lwt.2013.09.011.

Campos, E. V. R. et al. Use of botanical insecticides for sustainable agriculture: Future perspectives. Ecological Indicators, v. 105, p. 483-495, 2019.

Candiago, N. T.; Ansiliero, R.; Gelinski, J. L. N. Revisão sobre métodos de microencapsulação. Anuário Pesquisa e Extensão Unoesc Videira, v. 3, pp. e17281, 2018.

Carvalho, W.; Canilha, L.; Silva, S. S. D. Uso de biocatalisadores imobilizados: uma alternative para a condução de bioprocessos. Revista Analytica, Rio de Janeiro, pp. 60-70, 2006.

Castro, A. G. D.; Rodrigues, I. A química e a reologia no processamento dos alimentos. Lisboa, PO: Instituto Piaget, 2003.

Chandler, D. et al. Microbial biopesticides for integrated crop management: an assessment of environmental and regulatory sustainability. Journal of Food Science and Technology, Mysore, India, v. 19, p. 275-283, 2008. http://dx.doi.org/10.1016/ j.tifs.2007.12.009.

Cook, M. T. et al. Microencapsulation of probiotics for gastrointestinal delivery. Journal of Controlled Release, Amsterdam, v. 162, n. 1, p. 56-67, 2012. 
Copping, L.G.; Menn, J. J. Biopesticides: a review of their action, applications and efficacy. Pest Management Science, Sussex, v. 56, p. 651-676. 2000.

Da Silva, B. V.; Barreira, J. C.; Oliveira, M. B. P. Natural phytochemicals and probiotics as bioactive ingredients for functional foods: Extraction, biochemistry and protected-delivery technologies. Trends in Food Science \& Technology, Cambridge, v. 50, p. 144-158, 2016.

Dara, S. K. Microbial control of arthropod pests in small fruits and vegetables in temperate climate. In: Lacey, L.A. (Ed.), Microbial control of insect and mite pests: from theory to practice. London: Elsevier Inc., 2017. p. 209-221. https://doi.org/ 10.1016/B978-012-803527-6.00014-7.

Dunham, B. Microbial pesticides: A key role in the multinational portfolio. New Ag International, pp. 32-36. 2015.

Eckert, C. et al. Microencapsulation of Lactobacillus plantarum ATCC 8014 through spray drying and using dairy whey as wall materials. Lebensmittel-Wissenschaft + Technologie, v. 82, p. 176-183, 2017. http://dx.doi.org/10.1016/j.lwt.2017.04.045.

Eski, A. et al. A new biopesticide from a local Bacillus thuringiensis var. tenebrionis (Xd3) against alder leaf beetle (Coleoptera: Chrysomelidae). World Journal of Microbiology \& Biotechnology, Oxford, v. 33, n. 5, p. 95, 2017. http://dx.doi.org/10.1007/s11274-017-2263-0.

Eski, A.; Demirbağ, Z.; Demir, İ. Microencapsulation of an indigenous isolate of Bacillus thuringiensis by spray drying. Journal of Microencapsulation, London, v. 36, n. 1, p. 1-9, 2019.

Esser-Kahn, A. P. et al. Triggered release from polymer capsules. Macromolecules, Easton, v. 44, p. 5539-5553, 2011. http://dx.doi. org/10.1021/ma201014n.

Fernández-Pérez, M. et al. Lignin and lignosulfonate-based formulations to protect pyrethrins against photodegradation and volatilization. Industrial \& Engineering Chemistry Research, Washington, v. 53, p. 13557-13564, 2014. http://dx.doi.org/10.1021/ie500186e.

Fontaniella, B. et al. Identification of xanthans isolated from sugarcane juices obtained from scalded plants infected by Xanthomonas albilineans. Journal of Chromatography. B, Analytical Technologies in the Biomedical and Life Sciences, v. 770, n. 1-2, p. 275-281, 2002.

Galus, S.; Kadzińska, J. Food applications of emulsion-based edible films and coatings. Trends in Food Science \& Technology, Cambridge, v. 45, n. 2, p. 273-283, 2015.

García-Gutiérrez, K. et al. Small microcapsules of crystal proteins and spores of Bacillus thuringiensis by an emulsification/internal gelation method. Bioprocess and Biosystems Engineering, Berlin, v. 4, pp. 701-708, 2011.

Glare, T. et al. Have biopesticides come of age? Trends in Biotechnology, Amsterdam, v. 30, p. 250-258, 2012.

Glare, T. R.; O'Callaghan, M. Microbial biopesticides for control of invertebrates: progress from New Zealand. Journal of Invertebrate Pathology, San Diego, v. 165, p. 82-88, 2019. http://dx.doi. org/10.1016/j.jip.2017.11.014.

$\mathrm{He}$, X. et al. Biopolymer microencapsulations of Bacillus thuringiensis crystal preparations for increased stability and resistance to environmental stress. Applied Microbiology and Biotechnology, Berlin, v. 101, n. 7, p. 2779-2789, 2017.

Holkem, A. T.; Codevilla, C. F.; De Menezes, C. R. Emulsificação/ gelificação iônica interna: Alternativa para microencapsulação de compostos bioativos. Ciência e Natura, Santa Maria, v. 37, n. 5, p. 116-124, 2015 .

Kamel, S. et al. Pharmaceutical significance of cellulose: a review. Express Polymer Letters, Budapest, v. 2, n. 11, p. 758-778, 2008.
Khorramvatan, S. et al. The effect of polymers on the stability of microencapsulated formulations of Bacillus thuringiensis subsp. Kurstaki (Bt-KD2) after exposure to ultra violet radiation. Biocontrol Science and Technology, Oxford, v. 24, p. 215-220, 2014.

Koç, M. et al. Microencapsulation of Extra Virgin Olive Oil by Spray Drying: Effect of Wall Materials Composition, Process Conditions, and Emulsification Method. Food and Bioprocess Technology, v. 8, p. 301-318, 2015.

Lacey, L. A. et al. Insect pathogens as biological control agents: back to the future. Journal of Invertebrate Pathology, San Diego, v. 132, p. 1-41, 2015. http://dx.doi.org/10.1016/j.jip.2015.07.009.

Liu, C. P.; Liu, S. D. Formulation and characterization of the microencapsulated entomopathogenic fungus Metarhizium anisopliae MA126. Journal of Microencapsulation, London, v. 26, p. 377-384, 2009a.

Liu, C. P.; Liu, S. D. Low-temperature spray drying for the microencapsulation of the fungus Beauveria bassiana. Drying Technology, New York, v. 27, p. 747-753, 2009 b.

Mcclements, D. J.; Li, Y. Structured emulsion-based delivery systems: controlling the digestion and release of lipophilic food componentes. Advances in Colloid and Interface Science, Amsterdam, v. 159, p. 213-228, 2010. http://dx.doi.org/10.1016/j.cis.2010.06.010.

Mcmillin, K. W. Advancements in meat packaging. Meat Science, Barking, v. 132, p. 153-162, 2017.

Mortazavian, A. M. et al. Survival of encapsulated probiotic bacteria in iranian yogurt drink (doogh) after the product exposure to simulated gastrointestinal conditions. Milchwissenschaft. Milk Science International, Munchen, v. 63, n. 4, p. 427-429, 2008.

Muñoz-Celaya, A. L. et al. Spray-drying microencapsulation of Trichoderma harzianum conidias in carbohydrate polymers matrices. Carbohydrate Polymers, Barking, v. 88, p. 1141-1148, 2012.

Pereira, K. C. et al. Microencapsulação e liberação controlada por difusão de ingredientes alimentícios produzidos através da secagem por atomização: revisão. Brazilian Journal of Food Technology, Campinas, v. 21, p. 1-9, 2018.

Pérez-Landa, I. D. et al. Photoprotection and release study of spinosad biopolymeric microparticles obtained by spray drying. Powder Technology, Lausanne, v. 377, p. 514-522, 2021.

Perez-Masia, et al. Encapsulation of folic acid in food hydrocolloids through nanospray drying and electrospraying for nutraceutical applications. Food Chemistry, London, v. 168, p. 124-133, 2015. http://dx.doi.org/10.1016/j.foodchem.2014.07.051.

Phadungath, C. Casein micelle structure: a concise review. Journal of Science and Technology, London, v. 27, n. 1, p. 201-212, 2005.

Preininger, C. et al. Concepts and applications of foliar spray for microbial inoculants. Applied Microbiology and Biotechnology, Berlin, v. 102, p. 7265-7282, 2018. http://dx.doi.org/10.1007/ s00253-018-9173-4.

Putri, Y. R. P. et al. Study controlled release, toxicity test, and pesticide test of microcapsule eugenol with casein micelle. AIP Conference Proceedings, New York, v. 2085, n. 020015, 2019.

Ribeiro, E. P.; Seravalli, E. A. G. Química de Alimentos. Blücher: Instituto Mauá De Tecnologia, 2007.

Richards, E. H. et al. Novel control methods for insect pests: Development of a microencapsulation procedure for proteinaceous bioactives intended for oral delivery. Pest Management Science, Sussex, v. 71, p. 1238-1246, 2015.

Rodríguez, A. P. G. et al. Bio-insecticide Bacillus thuringiensis spores encapsulated with amaranth derivatized starches: studies on the 
propagation in vitro. Bioprocess and Biosystems Engineering, Berlin, v. 38, n. 2, p. 329-339, 2015. http://dx.doi.org/10.1007/ s00449-014-1273-7.

Shahidi, F.; Han, X. Q. Encapsulation of food ingredients. Critical Reviews in Food Science and Nutrition, Boca Raton, v. 33, n. 6, p. 501-547, 1993.

Sharma, R.; Goel, A. Development of insect repellent finish by a simple coacervation microencapsulation technique. International Journal of Clothing Science and Technology, United Kingdom, v. 30, n. 2, p. 152-158, 2018. http://dx.doi.org/10.1108/IJCST-02-2017-0022.

Shokri, Z. et al. Factors affecting viability of Bifidobacterium bifidum during spray drying. Daru: Journal of Faculty of Pharmacy, Tehran University of Medical Sciences, v. 23, n. 7, 2015. http:/ dx.doi.org/10.1186/s40199-014-0088-z.

Sohail, A. et al. Survivability of probiotics encapsulated in alginate gel microbeads using a novel impinging aerosols method. International Journal of Food Microbiology, Amsterdam, v. 145, n. 1, p. 162-168, 2011.

Sporleder, M.; Lacey, L. A. Biopesticides. In: ALYOKHIN, A.; VINCENT, C.; GIORDANENGO, P. (Eds.). Insect Pests of Potato. London: Elsevier Inc., 2013. p. 463-497. https://doi.org/10.1016/ B978-0-12-386895-4.00016-8.

Suave, J. et al. Microencapsulação: Inovação em diferentes áreas. Health and Environment Journal, Singapore, v. 7, p. 12-20, 2006.
Tamez-Guerra, P. et al. Storage stability of Anagrapha falcifera nucleopolyhedrovirus in spray-dried formulations. Journal of Invertebrate Pathology, San Diego, v. 79, p. 7-16, 2002.

Thakore, Y. The biopesticide market for global agricultural use. Industrial Biotechnology, New Rochelle, v. 2, p. 194-208, 2006.

Tomazelli Júnior, O. et al. Microencapsulation of essential thyme oil by spray drying and its antimicrobial evaluation against Vibrio alginolyticus and Vibrio parahaemolyticus. Brazilian Journal of Biology, v. 78, n. 2, p. 311-317, 2018. https://doi.org/10.1590/1519-6984.08716.

West, S. D. Determination of the naturally derived insect control agent Spinosad and its metabolites in soil, sediment, and water by HPLC with UV detection. Journal of Agricultural and Food Chemistry, Easton, v. 45, p. 3107-3113, 1997. http://dx.doi. org/10.1021/jf9701648.

Xing, Y. et al. Effect of porous starch concentrations on the microbiological characteristics of microencapsulated Lactobacillus Acidophilus. Food \& Function, Cambridge, v. 5, n. 5, p. 972-983, 2014.

Zaitoon, A.; Lim, L.; Scott-Dupree, C. Activated release of ethyl formate vapor from its precursor encapsulated in ethyl Cellulose/ Poly(Ethylene oxide) electrospun nonwovens intended for active packaging of fresh produce. Food Hydrocolloids, Oxford, v. 112, p. 106313, 2021. http://dx.doi.org/10.1016/j.foodhyd.2020.106313.

Recebido: 31 mar. 2021 Aprovado: 25 maio 2021 